\title{
Prophylactic Biliary Stenting Before Cholecystectomy in Patients With Gallstones and Common Bile Duct Stones
}

\author{
Hideaki Kawabata $^{\text {a, c }, \text { Yukino Kawakatsu }}{ }^{\mathrm{a}}$, Katsutoshi Yamaguchia ${ }^{\mathrm{a}}$, Daiki Sone ${ }^{\mathrm{a}}$, Naonori Inoue ${ }^{\mathrm{a}}$, \\ Yuki Ueda ${ }^{\text {a }}$, Yuji Okazakia , Misuzu Hitomi ${ }^{\mathrm{a}}$, Masatoshi Miyata ${ }^{\mathrm{a}}$, Shigehiro Motoi ${ }^{\mathrm{a}}$, \\ Kenichirou Fukuda $^{b}$, Yoshihiro Shimizu ${ }^{b}$
}

\begin{abstract}
Background: The usefulness of prophylactic biliary stenting for patients with common bile duct stones (CBDS) and gallstones (GS) to prevent recurrent biliary events after endoscopic sphincterotomy (EST) and CBDS extraction before elective cholecystectomy remains controversial. The aim of this study was to evaluate the risk of recurrent CBDS around the perioperative period and clarify its risk factors.
\end{abstract}

Methods: The clinical data of all patients who received prophylactic biliary stenting after EST for CBDS and later underwent cholecystectomy for GS followed by stent extraction in our institution were retrospectively reviewed. The numbers of residual CBDS at the end first and second endoscopic retrograde cholangiography (ERC) studies were compared. Univariate and multivariate analyses were performed using a logistic regression model to determine risk factors for recurrent CBDS in the perioperative period.

Results: Forty-two consecutive patients received prophylactic biliary stenting and subsequent cholecystectomy for GS. Three of these patients were excluded from this study because the number of residual stones was not confirmed. The median maximum CBDS diameter at second ERC was $0 \mathrm{~mm}$ (range, $0-10 \mathrm{~mm}$ ); six patients had multiple CBDS $(\geq 5)$. The number of CBDS at second ERC was increased in comparison to that at the first ERC in 15 patients (38.4\%), and was unchanged or decreased in 24 patients. The median minimum cystic duct diameter was $4 \mathrm{~mm}$ (range, $1-8 \mathrm{~mm}$ ). The median interval between first ERC and operation was 26 days (range, 2 - 131 days). The median interval between operation and second ERC was 41 days (range, 26 - 96 days). Laparoscopic cholecystectomy (LC) was performed in 38 patients, one of whom was converted from LC to open cholecystectomy. Postoperative complications (transient bacteremia) occurred in one patient. The cystic duct diameter was an independ-

Manuscript submitted July 24, 2019, accepted August 13, 2019

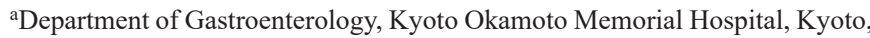
Japan

bepartment of Surgery, Kyoto Okamoto Memorial Hospital, Kyoto, Japan

${ }^{c}$ Corresponding Author: Hideaki Kawabata, Department of Gastroenterology, Kyoto Okamoto Memorial Hospital, 100 Nishinokuchi, Sayama, Kumiyamacho, Kuze-gun, Kyoto 613-0034, Japan. Email: hkawabata@okamoto-hp.or.jp

doi: https://doi.org/10.14740/gr1207 ent risk factor for an increased number of CBDS at second ERC in the multivariate analysis (odds ratio 0.611 (95\% confidence interval (0.398 - 0.939)), $\mathrm{P}=0.03)$.

Conclusion: Recurrent CBDS around the perioperative period of cholecystectomy is not a rare complication after EST and the removal of CBDS with concomitant GS. Prophylactic biliary stenting is considered useful for preventing CBDS-associated complications, especially for patients in whom the cystic duct diameter is larger $(\geq$ $5 \mathrm{~mm})$.

Keywords: CBD stone; Gallstone; Biliary stent; Endoscopic sphincterotomy; Cholecystectomy

\section{Introduction}

Endoscopic sphincterotomy (EST) and stone extraction have been accepted in the standard management of common bile duct stones (CBDS) [1-3]. In patients with concomitant gallstones (GS), elective laparoscopic cholecystectomy (LC) after EST and stone extraction is recommended because it reduces the occurrence of recurrent biliary events from $21-47 \%$ to $2-7 \%$, including cholecystitis, cholangitis, pancreatitis, jaundice and biliary colic [4-11]. In another retrospective analysis, $20 \%$ of all post-EST patients had recurrent biliary complications during the waiting period for cholecystectomy, the median time of which was 22 days [12]. Thus, some clinicians perform prophylactic CBD stenting to prevent recurrent biliary events due to GS entering the CBD around the perioperative period. However, one study that retrospectively and prospectively compared groups of patients with CBDS who underwent EST with or without prophylactic CBD stent insertion before cholecystectomy concluded that prophylactic stent insertion had no impact on biliary complications [13].

The correlation between prophylactic biliary stenting and the outcomes of cholecystectomy, such as the rates of conversion from LC to open cholecystectomy (OC) and postoperative complications remains controversial. An experimental study in dogs revealed that 4 weeks of biliary stenting resulted in bile duct fibrosis, with severe chronic inflammation and papillary hyperplasia of the epithelium, while bile cultures grew fecal bacteria [14]. Moreover, stenting and subsequent surgi- 


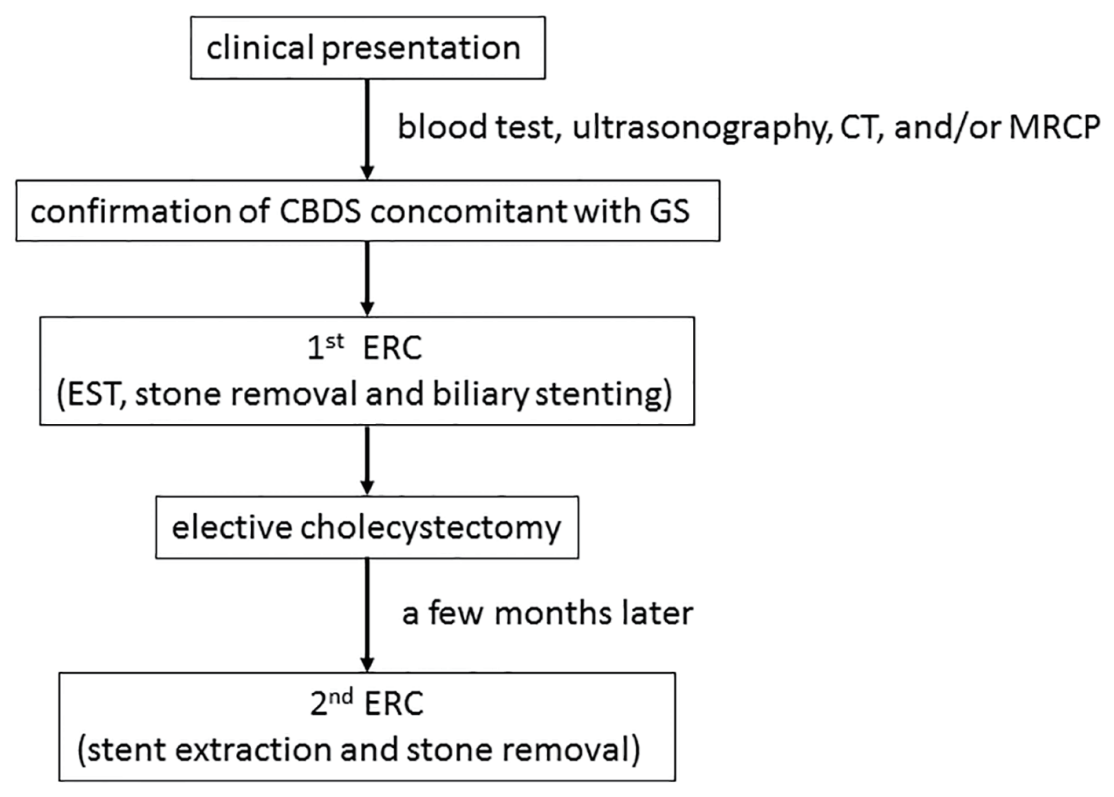

Figure 1. A flow chart of the clinical strategy for the management of CBDS concomitant with GS in the present study. CBDS: common bile duct stones; GS: gallstones; ERC: endoscopic retrograde cholangiography; EST: endoscopic sphincterotomy; CT: computed tomography; MRCP: magnetic resonance cholangiopancreatography.

cal treatment resulted in a higher incidence of postoperative complications. Nair et al [15] suggested that the placement of a bile duct stent for long duration before elective LC affected the operation time, conversion rate, bile leak rate and length of hospital stay. In contrast, Lee et al [16] reported there were no surgical complications regardless of whether biliary stenting was performed, and that the insertion of a stent was not a predictor of conversion to $\mathrm{OC}$ or a long operative time.

The aim of this study was to evaluate the risk of recurrent CBDS around the perioperative period and clarify its risk factors. Thus, the present study discusses the utility of prophylactic biliary stenting before cholecystectomy in patients with GS and CBDS.

\section{Materials and Methods}

We retrospectively reviewed the clinical data of all patients who received prophylactic biliary stenting after EST and endoscopic stone removal for CBDS and who later underwent cholecystectomy for GS followed by stent extraction in our institution between August 2016 and May 2018.

All consecutive patients diagnosed as CBDS concomitant with GS underwent EST, stone extraction for CBDS and biliary stenting using a 7-Fr pigtail plastic stent (Zimmon Biliary Stent, Cook Medical, Tokyo, Japan). Then, elective cholecystectomy was performed after the patients recovered to a good condition. A few months after cholecystectomy, we extracted the stent, confirmed the presence of residual CBDS by endoscopic retrograde cholangiography (ERC) and then removed them completely (Fig. 1).

The variables collected included gender, age, maximum diameter of GS and CBDS, patients with multiple GBS and
CBDS ( $\geq 5$ ), cholangitis on admission, minimum cystic duct diameter, residual CBDS at first ERC, duration between first ERC and operation, LC or OC, conversion rate, operation time, postoperative complications and duration between operation and second ERC. We compared the number of residual CBDS at the end of first ERC with that at the start of second ERC. The existence and diameter of GS were confirmed by ultrasonography, computed tomography (CT), magnetic resonance cholangiopancreatography (MRCP), and/or ERC. The diameters of CBD, CBDS and cystic duct were measured on ERC images. Cholangitis was diagnosed according to Tokyo Guidelines 2018 [17].

All patients provided their written informed consent. This study followed the ethical guidelines for studies involving human subjects based on the Helsinki Declaration. The study protocol was approved by the institutional review board of Kyoto Okamoto Memorial Hospital.

Statistical analyses were performed using the SPSS software program (version 24.0, IBM SPSS). Categorical variables were compared using Fisher's exact test. Continuous variables were expressed as the median (range) and were compared using Student's $t$-test. The level of significance was set to $\mathrm{P}<0.05$. Univariate and multivariate analyses with a logistic regression model were performed to determine the risk factors for recurrent $\mathrm{CBDS}$ in the perioperative period. In the multivariate analysis, parameters with $\mathrm{P}$ values of $<0.2$ in the univariate analyses were entered into the logistic regression model.

\section{Results}

Forty-two consecutive patients received prophylactic biliary 
Table 1. Patient Characteristics According to the Change in the Number of CBDS Between First and Second ERC

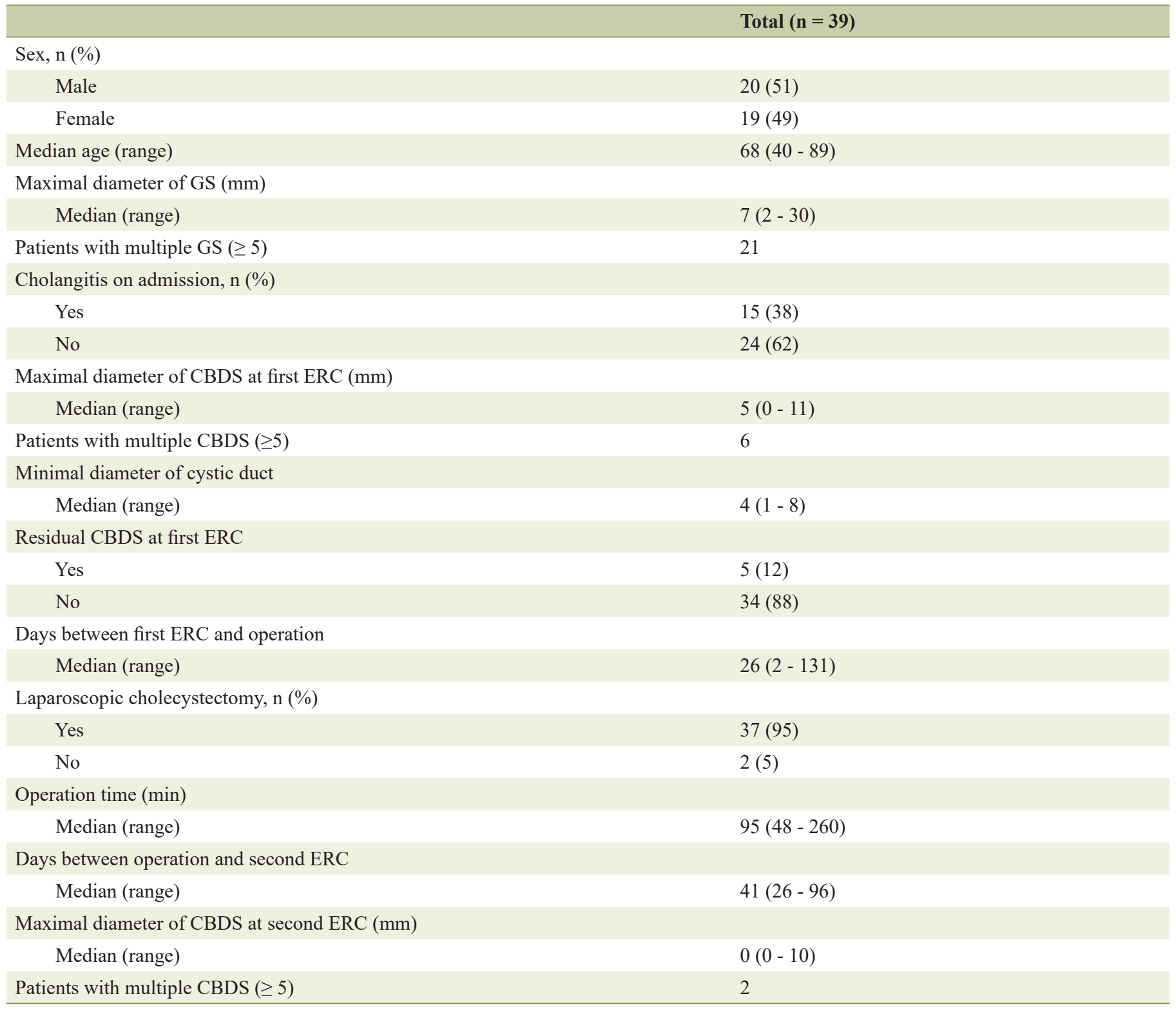

GS: gallstones; CBDS: common bile duct stones; ERC: endoscopic retrograde cholangiography.

stenting after EST and endoscopic stone removal for CBDS and subsequent cholecystectomy for GS in our institution during the study period. Three of these patients were excluded from the study because the number of residual stones was not confirmed radiographically during first ERC due to severe cholangitis.

The patient characteristics are outlined in Table 1. The study population included 20 males and 19 females with a median age of 68 years (range, 40 - 89 years). The median maximum GS diameter was $7 \mathrm{~mm}$ (range, 2 - $30 \mathrm{~mm}$ ), and 21 patients had multiple CBDS $(\geq 5)$. Whereas, the median maximum CBDS diameter at first ERC was $5 \mathrm{~mm}$ (range, $0-11$ $\mathrm{mm}$ ), and six patients had multiple CBDS ( $\geq 5)$. In contrast, no CBDS were detected radiographically by first ERC in six patients despite the existence of CBDS in other preoperative images. Fifteen patients were diagnosed with cholangitis on admission. The median minimum cystic duct diameter was 4 $\mathrm{mm}$ (range, $1-8 \mathrm{~mm}$ ). CBDS were not completely removed at first ERC in five patients $(12 \%)$, all of whom had one residual stone. LC was performed in 38 patients, one of whom was converted from LC to $\mathrm{OC}$ due to a laparoscopic finding of cystic duct necrosis, which had probably been caused by a previous episode of cholecystitis. In contrast, OS was performed in one patient because of a past history of abdominal surgery with unknown origin. The median operation time was $95 \mathrm{~min}$ (range, 48 - $260 \mathrm{~min}$ ). Transient bacteremia, without any findings of cholangitis, occurred after LC in one patient. The median interval between first ERC and the operation was 
Table 2. Patient Characteristics According to Change in the Number of CBDS Between First and Second ERC

\begin{tabular}{llll}
\hline \multirow{2}{*}{ Variables } & \multicolumn{2}{c}{ Change in number of CBDS between first and second ERC } \\
\cline { 2 - 3 } & Increased (group A) $(\mathbf{n = 1 5})$ & Unchanged or decreased (group B) (n= 24) \\
\hline Gender, female & $7(46.6 \%)$ & $12(50 \%)$ & 0.55 \\
Age, years & $71(40-89)$ & $66(45-84)$ & 0.211 \\
Maximum diameter of GS, mm & $7(2-22)$ & $7(2-30)$ & 0.233 \\
Patients with multiple GS $(\geq 5)$ & $9(60 \%)$ & $12(50 \%)$ & $10(41.6 \%)$ \\
Cholangitis on admission & $5(33.3 \%)$ & $5(0-11)$ & 0.55 \\
Maximum diameter of CBDS, mm & $4(0-11)$ & $2(8.3 \%)$ & $3(1-7)$ \\
Patients with multiple CBDS $\geq 5)$ & $4(26.6 \%)$ & $5(20.8 \%)$ & 0.43 \\
Minimal diameter of cystic duct, mm & $5(1-8)$ & $23(2-97)$ & 0.139 \\
Residual CBDS at first ERC & $0(0 \%)$ & $24(100 \%)$ \\
Days between first ERC and operation & $28(12-131)$ & $94(48-260)$ \\
Laparoscopic cholecystectomy & $13(86.6 \%)$ & $40(26-96)$ \\
\hline Operation time, min & $95(56-163)$ & 0.074 \\
Days between operation and second ERC & $44(31-92)$ & 0.176 \\
\hline
\end{tabular}

Results are presented as the number $(n)$ or mean $n(\%)$ for qualitative data or as the median (range) for quantitative data. * $<0.05$. GS: gallstones; CBDS: common bile duct stones; ERC: endoscopic retrograde cholangiography.

26 days (range, 2 - 131 days). The median interval between the operation and second ERC was 41 days (range, 26 - 96 days).

At second ERC, the median maximum CBDS diameter was $0 \mathrm{~mm}$ (range, $0-10 \mathrm{~mm}$ ), and six patients had multiple CBDS $(\geq 5)$. Residual stones were completely removed in all patients, after which they remained stent-free. The number of CBDS at second ERC was increased in comparison to that at the end of first ERC in 15 patients (38.4\%, group A), whereas it was unchanged or decreased in 24 patients (group B) (unchanged, $n=23$; decreased, $n=1$ ).

After first ERC, four patients developed mild pancreatitis, which was treated with conservative therapy. Three patients developed post-EST bleeding, requiring endoscopic hemostasis. After second ERC, a subcapsular hepatic hematoma, which required an operation, occurred in one patient, and one patient suffered anaphylaxis due to antibiotics, which was treated by conservative therapy.

Table 2 compares group A and group B. The minimal cystic duct diameter was significantly larger in group $\mathrm{A}(\mathrm{P}=$ 0.022). No significant differences were observed in any other factors $(\mathrm{P}>0.05)$.

Table 3 shows the results of the univariate and multivariate analyses. In the univariate analysis, the cystic duct diameter was correlated with an increase number of CBDS at second ERC (odds ratio (OS) 0.664 (95\% confidence interval (CI) 0.459 - 0.962), $\mathrm{P}=0.03$ ). Multiple CBDS $(\geq 5)$ and long duration between first ERC and the operation both had $\mathrm{P}$ values of $<0.20$ and were included in the multivariate analysis $(\mathrm{P}=0.141$ and $\mathrm{P}=0.185$, respectively). In the multivariate analysis, which included these three factors, the cystic duct diameter was an independent risk factor for an increased number of CBDS at second ERC, after excluding other confounding factors (OR 0.611 (95\% CI (0.398 - 0.939)), $\mathrm{P}=$
$0.03)$

\section{Discussion}

We showed that recurrent CBDS around the perioperative period of cholecystectomy was not a rare complication and suggested that prophylactic biliary stenting was useful for preventing CBDS-associated cholangitis. In the patients with an increased number of CBDS at second ERC, there were three periods in which recurrent CBDS developed: when GS entered the CBD between first ERC and cholecystectomy, when GS entered the CBD during cholecystectomy and when CBDS developed between cholecystectomy and second ERC. Among these periods, the development of CBDS between cholecystectomy and second ERC can be excluded because some reports have described that CBDS decreased in size and diameter, or disappeared in the majority of patients with biliary stent placement after EST [18, 19]. Moreover, it is unlikely for GS to enter the CBD during cholecystectomy because of small number of CBDS-associated complications reported after surgery $[4,12,15,16]$. Thus, in the present study, recurrent CBDS is likely to have developed between first ERC and cholecystectomy, namely, during the waiting period for cholecystectomy in almost all patients. In a prospective randomized study, 14\% of patients presented with cholangitis with a median followup period of approximately 5 years after a wait-and-see policy after endoscopic stone removal for patients with GS [4]. As mentioned above, in a retrospective study, $20 \%$ of all post-EST patients had recurrent biliary complications including recurrent CBDS (5\%) and cholangitis (2\%) during the waiting period for cholecystectomy, the median time of which was 22 days [12]. In the present study, the number of CBDS at second ERC in comparison to that at the end of first ERC was increased in 
Table 3. Factors Associated With the Development of Recurrent CBDS After Endoscopic Treatment for CBDS Around the Perioperative Period in Cholecystectomy

\begin{tabular}{|c|c|c|c|c|}
\hline & Univariate OR (95\% CI) & P value & Multivariate OR (95\% CI) & P value \\
\hline Gender, female & $1.143(0.314-4.160)$ & 0.839 & & \\
\hline Age, years & $0.961(0.903-1.023)$ & 0.211 & & \\
\hline Patients with multiple GS ( $\geq 5$ ) & $1.143(0.314-4.160)$ & 0.839 & & \\
\hline Cholangitis on admission & $1.429(0.372-5.487)$ & 0.603 & & \\
\hline Patients with multiple CBDS $(\geq 5)$ & $0.250(0.039-1.582)$ & 0.141 & $0.218(0.030-1.586)$ & 0.132 \\
\hline Minimal diameter of cystic duct, $\mathrm{mm}$ & $0.664(0.459-0.962)$ & $0.03 *$ & $0.611(0.398-0.939)$ & $0.025^{*}$ \\
\hline Residual CBDS at first ERC & & 0.999 & & \\
\hline Days between first ERC and operation & $0.984(0.962-1.008)$ & 0.185 & $0.974(0.946-1.003)$ & 0.084 \\
\hline Laparoscopic cholecystectomy & & 0.999 & & \\
\hline
\end{tabular}

Results are presented as a number $(\mathrm{n})$ or mean $\mathrm{n}(\%)$ for qualitative data or as median (range) for quantitative data. ${ }^{*} \mathrm{P}<0.05 . \mathrm{GS}$ : gallstones; CBDS: common bile duct stones; ERC: endoscopic retrograde cholangiography; $\mathrm{Cl}$ : confidence interval.

$38 \%$ of patients with a median interval of 26 days; this rate of recurrent CBDS was higher in comparison to previous studies. One possible reason for this discrepancy was that the number of patients with recurrent CBDS in previous studies may have been underestimated, because patients with subclinical recurrent CBDS were not included. In addition, a few patients in the present study may have developed recurrent CBDS during cholecystectomy.

The correlation between prophylactic biliary stenting and the outcome of cholecystectomy remains controversial as mentioned above. In the present study, the rate of conversion to $\mathrm{OC}$ in patients who underwent LC was $2 \%$, which was considerably lower in comparison to those in previous study on prophylactic biliary stenting (51\%) [15]. Moreover, we experienced fewer postoperative complications and there was no mortality. The short waiting period for cholecystectomy (median 26 days, range, 2 - 141 days) may have contributed to this favorable outcome, because a previous study showed that long biliary stent placement (1 month) induced both chronic inflammation and bacterial growth [14], and another study suggested that a longer waiting period (mean 149 days; range, 8 - 400 days) affected postoperative outcomes [15]. Furthermore, one retrospective study investigating the optimal timing of elective LC after acute cholangitis and subsequent clearance of CBDS showed that both late surgery ( $>$ 6 weeks) and a history of EST were independent risk factors for postoperative complications in a multivariate analysis [20]. Thus, early elective LC, as soon as the patient recovers to a good condition, should be recommended after EST for CBDS, irrespective of performance of biliary stenting in order to prevent postoperative complications. In addition, prophylactic stents should be removed promptly after cholecystectomy.

We also showed that the diameter of the cystic duct was an independent risk factor for an increased number of CBDS at second $\mathrm{ERC}$ in a multivariate analysis. It is understandable that when the diameter of the cystic duct is larger, GS can enter the CBD more easily. This is the first report to investigate and elucidate the correlation between the cystic duct diameter and recurrent CBDS. Thus, it may be reasonable to restrict prophylactic $\mathrm{CBD}$ stenting to patients, in whom the minimum diameter of the cystic duct is larger $(\geq 5 \mathrm{~mm})$, based on the potential for inflammatory change of the CBD due to biliary stenting, the risk of repeated ERC and medical economy.

The present study is associated with some limitations. First, it was a retrospective single-arm study with a relatively small population. Larger, prospective, randomized trials are needed to confirm the utility of prophylactic CBD stenting in the perioperative period and to elucidate the risk factors for recurrent CBDS. Second, we were not able to accurately classify the phases in which recurrent CBDS occurred. The times were classified into the waiting time for cholecystectomy, during cholecystectomy and after cholecystectomy; however, in most cases, it is likely that recurrent CBDS would have developed while the patients were waiting for cholecystectomy. To clarify incidence of recurrent CBD in each phase would contribute to the development of detailed strategies for the management of CBDS concomitant with GS.

\section{Conclusion}

Recurrent CBDS around the perioperative period of cholecystectomy is not a rare complication in patients who have undergone EST and endoscopic removal of CBDS concomitant with GS, and prophylactic biliary stenting is considered to be useful for preventing CBDS-associated complications, especially in 
cases in which the cystic duct has a larger diameter.

\section{Acknowledgments}

None to declare.

\section{Financial Disclosure}

None to declare.

\section{Conflict of Interest}

The authors declare that they have no conflict of interest.

\section{Informed Consent}

Written informed consent was obtained from the patient.

\section{Author Contributions}

H. Kawabata, Y. Kawakatsu, K. Yamaguchi, D. Sone, N. Inoue, Y. Ueda, Y. Okazaki, M. Hitomi and M. Miyata contributed to endoscopic diagnosis and treatment. K. Fukuda and Y. Shimizu contributed to surgical treatment. M. Miyata and S. Motoi supervised the findings of this work. H. Kawabata wrote the manuscript with support from M. Hitomi and S. Motoi. All authors discussed the results and contributed to the final manuscript.

\section{References}

1. Kawai K, Akasaka Y, Murakami K, Tada M, Koli Y. Endoscopic sphincterotomy of the ampulla of Vater. Gastrointest Endosc. 1974;20(4):148-151.

2. Nakajima M, Kimoto K, Fukumoto K, Ikehara H, Kawai K. Endoscopic sphincterotomy of the ampulla of Vater and removal of common duct stones. Am J Gastroenterol. 1975;64(1):34-43.

3. Cotton PB, Geenen JE, Sherman S, Cunningham JT, Howell DA, Carr-Locke DL, Nickl NJ, et al. Endoscopic sphincterotomy for stones by experts is safe, even in younger patients with normal ducts. Ann Surg. 1998;227(2):201-204.

4. Lau JY, Leow CK, Fung TM, Suen BY, Yu LM, Lai PB, Lam YH, et al. Cholecystectomy or gallbladder in situ after endoscopic sphincterotomy and bile duct stone removal in Chinese patients. Gastroenterology. 2006;130(1):96103.

5. Mador BD, Nathens AB, Xiong W, Panton ONM, Hameed SM. Timing of cholecystectomy following endoscopic sphincterotomy: a population-based study. Surg Endosc. 2017;31(7):2977-2985.
6. Tsai TJ, Lai KH, Lin CK, Chan HH, Wang EM, Tsai WL, Cheng JS, et al. The relationship between gallbladder status and recurrent biliary complications in patients with choledocholithiasis following endoscopic treatment. J Chin Med Assoc. 2012;75(11):560-566.

7. Boerma D, Rauws EA, Keulemans YC, Janssen IM, Bolwerk CJ, Timmer R, Boerma EJ, et al. Wait-and-see policy or laparoscopic cholecystectomy after endoscopic sphincterotomy for bile-duct stones: a randomised trial. Lancet. 2002;360(9335):761-765.

8. Barkun J, Dixon E, Strasberg S, Evidence Based Reviews In Surgery G. Canadian Association of General Surgeons and American College of Surgeons evidence based reviews in surgery. 13. Wait-and-see policy or laparoscopic cholecystectomy after endoscopic sphincterotomy for bile-duct stones. Can J Surg. 2005;48(3):244-246.

9. Hammarstrom LE, Holmin T, Stridbeck H, Ihse I. Longterm follow-up of a prospective randomized study of endoscopic versus surgical treatment of bile duct calculi in patients with gallbladder in situ. Br J Surg. 1995;82(11):1516-1521.

10. Targarona EM, Ayuso RM, Bordas JM, Ros E, Pros I, Martinez J, Teres J, et al. Randomised trial of endoscopic sphincterotomy with gallbladder left in situ versus open surgery for common bileduct calculi in high-risk patients. Lancet. 1996;347(9006):926-929.

11. Manes G, Paspatis G, Aabakken L, Anderloni A, Arvanitakis M, Ah-Soune P, Barthet M, et al. Endoscopic management of common bile duct stones: European Society of Gastrointestinal Endoscopy (ESGE) guideline. Endoscopy. 2019;51(5):472-491.

12. Schiphorst AH, Besselink MG, Boerma D, Timmer R, Wiezer MJ, van Erpecum KJ, Broeders IA, et al. Timing of cholecystectomy after endoscopic sphincterotomy for common bile duct stones. Surg Endosc. 2008;22(9):20462050 .

13. Verzhbitsky V, Zeina AR, Depsames R, Ovadia B, Atia O, Fireman Z. Does prophylactic stent insertion to the common bile duct during endoscopic retrograde cholangiopancreatography (ERCP) before cholecystectomy have any impact on the rate of biliary complications? Surg Endosc. 2013;27(12):4620-4624.

14. Karsten TM, Davids PH, van Gulik TM, Bosma A, Tytgat GN, Klopper PJ, van der Hyde MN. Effects of biliary endoprostheses on the extrahepatic bile ducts in relation to subsequent operation of the biliary tract. J Am Coll Surg. 1994;178(4):343-352.

15. Nair MS, Uzzaman MM, Fafemi O, Athow A. Elective laparoscopic cholecystectomy in the presence of common bile duct stent. Surg Endosc. 2011;25(2):429-436.

16. Lee R, Ha H, Han YS, Jung MK, Chun JM. Predictive factors for long operative duration in patients undergoing laparoscopic cholecystectomy after endoscopic retrograde cholangiography for combined choledochocystolithiasis. Surg Laparosc Endosc Percutan Tech. 2017;27(6):491496.

17. Kiriyama S, Kozaka K, Takada T, Strasberg SM, Pitt HA, Gabata T, Hata J, et al. Tokyo Guidelines 2018: diagnostic criteria and severity grading of acute cholangitis (with 
videos). J Hepatobiliary Pancreat Sci. 2018;25(1):17-30.

18. Fan Z, Hawes R, Lawrence C, Zhang X, Zhang X, Lv W. Analysis of plastic stents in the treatment of large common bile duct stones in 45 patients. Dig Endosc. 2011;23(1):86-90.

19. Jain SK, Stein R, Bhuva M, Goldberg MJ. Pigtail stents: an alternative in the treatment of difficult bile duct stones. Gastrointest Endosc. 2000;52(4):490-493.

20. Li VK, Yum JL, Yeung YP. Optimal timing of elective laparoscopic cholecystectomy after acute cholangitis and subsequent clearance of choledocholithiasis. Am J Surg. 2010;200(4):483-488. 